\title{
ISS ECLSS Technology Evolution for Exploration
}

\author{
Robyn L. Carrasquillo \\ NASA Marshall Space Flight Center, Huntsville, AL, 35812
}

\begin{abstract}
The baseline environmental control and life support systems (ECLSS) currentiy deployed on the International Space Station (ISS) and the regenerative oxygen generation and water reclamation systems to be added in 2008 are based on technologies selected during the earty 1990's. While they are generally meeting, or exceeding requirements for supporting the ISS crew, lessons learned from hardware development and on orbit experience, together with advances in technology state of the art, and tite unique requirements for future manned exploration missions prompt consideration of the next steps to be taken to evolve these technologies to improve robustness and reliability, enhance performance, and reduce resource requirements such as power and logistics upmass. This paper discusses the current state of ISS ECLSS technology and identifies possible areas for evolutionary enhancement or improvement.
\end{abstract}

\section{Introduction}

The International Space Station (ISS) as currently constructed consists of a multi-element complex capable of supporting a permanent crew of three astronauts. By the Assembly Complete milestone, permanent crews of greater than three may be accommodated through the addition of new modules and environmental control and life support (ECLS) equipment. Figure 1 shows the planned distribution of the ECLS System at Assembly Complete.

Although the technology level of the Environmental Control and Life Support System (ECLSS) existing and planned for the ISS surpasses all previous manned spaceflight missions, there is room for improvement and further evolution/advancement to achieve an ECLSS that will meet NASA's needs for future exploration. The need to achieve greater independence from resupply of ground resources, as well as minimize use of on-orbit resources, is apparent. While the degree of oxygen and water loop closure is a key element for achieving long-duration selfsufficiency, such advances are not all that is needed. Robust components immune to failures that require parts replacement, and eliminating dependence on limited-life or expendable components, are also extremely important factors. The goal of developing self-sufficient ECLS technologies of the future must be pursued with careful and balanced consideration of both performance and robustness. Development and operational experience with the baseline ISS ECLSS gives insight into some suggested areas of improvement with these factors in mind. In the President's Vision for U.S. Space Exploration, life support is recognized as a key enabling technology. The challenge for NASA's life support community will be to effectively apply lessons learned from past development and operational experience to the next generation technologies.

\section{Description of the ISS ECLSS with Strengths and Weaknesses}

As shown in Figure 1, the ISS ECLSS is distributed throughout all elements. Both U.S. and Russian systems are utilized, and in many cases provide redundancy for each other. A brief description of each subsystem area follows along with associated strengths and weaknesses.

\section{A. Temperature and Humidity Control}

Temperature and Humidity Control is provided through ventilation fans, a low-temperature cooled hydrophilic-coated condensing heat exchanger $(\mathrm{CHX})$ /slurper, and rotary liquid separator to remove condensate from the air stream. Advantages of this technology include its long flight history, relative simplicity, and gravity insensitivity. However, on-orbit data indicates a potential problem with sloughing of the CHX coating material and subsequent liquid carryover. Also, the required low-temperature coolant complicates the associated thermal control system and carries insulation penalties. Particulate and airborne microbial control is provided through HEPA filters in the return air ducts. While this is a simple solution, the filters are expendable and require routine replacement. 


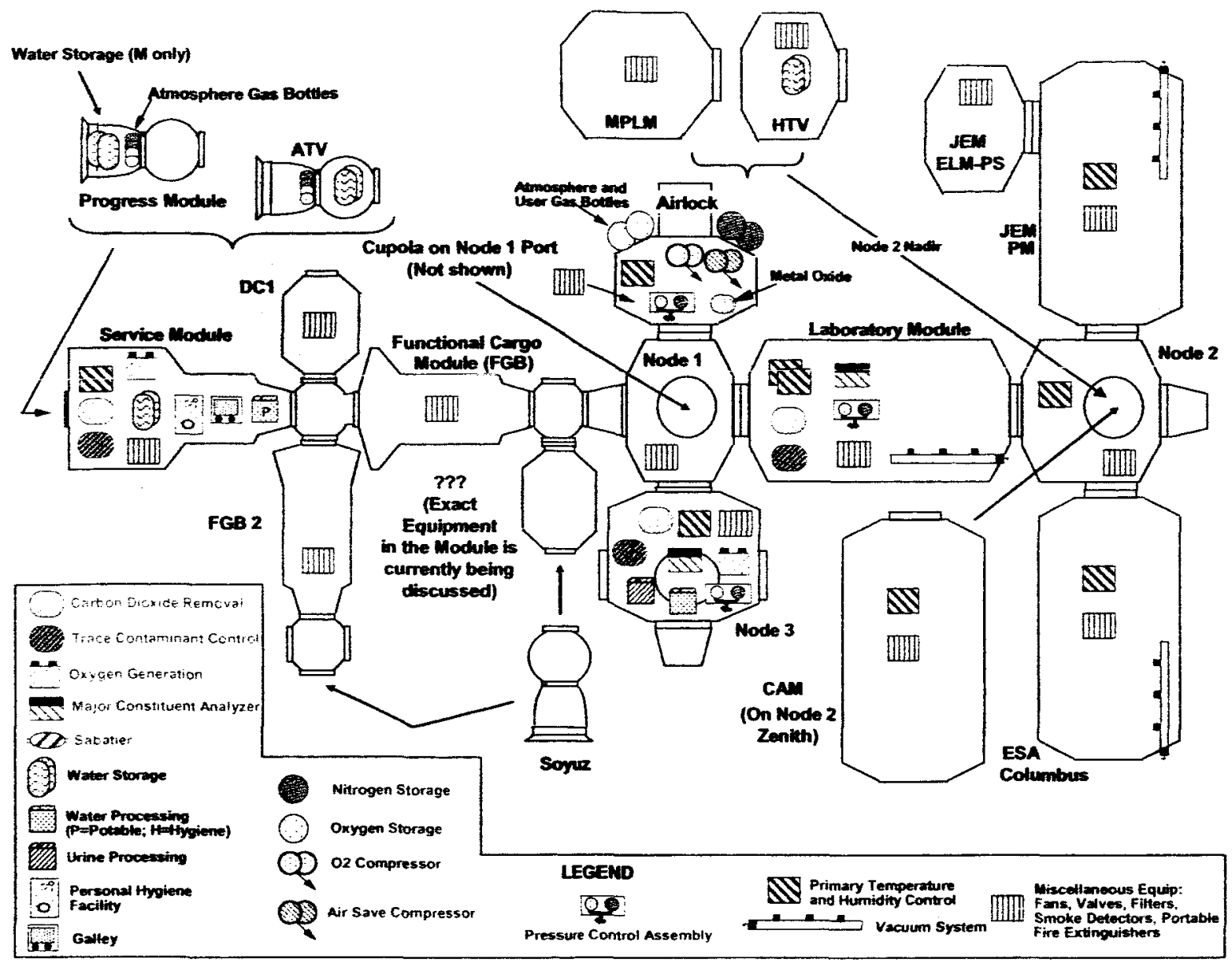

Figure 1. ISS Environmental Control and Life Support System Distribution at Assembly Complete

\section{B. Atmosphere Revitalization}

Carbon Dioxide Removal is provided by a 4-bed molecular sieve, containing zeolite for $\mathrm{CO}_{2}$ removal and a combination of zeolite and silica gel to desiccate the incoming air. Interstitial adsorbed air and desiccated water are returned to the cabin while the $\mathrm{CO}_{2}$ is desorbed to space vacuum with the aid of temperature. The Carbon Dioxide Removal Assembly's (CDRA's) advantages include water and air-save features, flight-proven history on multiple programs, and better than spec $\mathrm{CO}_{2}$ removal performance. It is fairly power-intensive and complex, and in its on-orbit $I S S$ performance to date it has encountered difficulties with zeolite particle dusting and contamination of downstream components.

The ISS Trace Contaminant Control System (TCCS) consists of an activated charcoal adsorption bed and thermal catalytic oxidizer with post-sorbent bed. The system is simple, provides broad spectrum contaminant control, and has encountered no significant on-orbit problems. The activated carbon bed is an expendable which requires routine replacement (projected to be 2.25 years based on 6-crew ISS contaminant loads) ${ }^{1}$, and the current catalyst must be protected from poisoning.

The chief atmosphere monitor on ISS is the Major Constituent Analyzer (MCA) which is based on mass spectrometry. While it has exhibited a stable, repeatable performance for analyzing $\mathrm{N}_{2}, \mathrm{O}_{2}, \mathrm{CO}_{2}, \mathrm{CH}_{4}, \mathrm{H}_{2}$, and $\mathrm{H}_{2} \mathrm{O}$, it has a number of drawbacks. It has a fairly complex control system, requires a roughing vacuum resource for startup, and the ion pump has a limited life. Its assay is limited to the six analytes listed above.

The Oxygen Generation Assembly (OGA) planned for, but not yet operational on, the ISS is a water electrolysis process that employs a solid polymer electrolyte. This is a well-characterized technology used on 
naval submarines. Its drawbacks include high power (by nature of the electrolysis process) and production of oxygen is limited to ambient pressure which makes it unable to be utilized to repressurize backup oxygen storage tanks.

The baseline ISS air revitalization system does not include a carbon dioxide reduction assembly due to NASA funding constraints, but scars are included in the Oxygen Generation System rack intended for later incorporation of such an assembly based on the Sabatier technology. Ground development of the Sabatier has advanced this technology to a Technology Readiness Level (TRL) of 4-5.

\section{Water Recovery and Management}

The planned ISS Water Recovery System (WRS) consists of urine processor and water processor assemblies. The urine processor is based on the Vapor Compression Distillation (VCD) technology, a phase change process which recovers water from urine through low-pressure evaporation in a rotating distillation assembly. This technology was successfully demonstrated in microgravity on STS-107 and is therefore at a TRL-7. ${ }^{3}$ Qual and acceptance testing of the ISS flight hardware is underway at the Marshall Space Flight Center (MSFC). It has the advantage of being a fairly power-efficient process, but is admittedly a complex mechanical design and is limited to a water recovery efficiency from urine of $85 \%$.

The ISS water processor produces potable water from a wastewater stream which includes urine distillate from the VCD, cabin air humidity condensate, and other hygiene and miscellaneous waste waters. The technology uses includes particulate filtration, adsorption and ion exchange, and heterogeneous catalytic oxidation, and employs rotary and membrane-based gas/liquid separators. This combination of technologies has been proven through many years of development testing and is robust in handling complex multiphase flow in microgravity. Drawbacks include expendable bed replacement and associated logistics penalties, and the higher power and complexity associated with the high-temperature catalytic reaction process. The catalytic reactor has also been susceptible to generation of particulates which can impact valves and other components; a soft-stow launch of this component and subsequent flushing are planned to mitigate this risk.

\section{Fire Detection and Suppression}

The ISS Fire Detection and Suppression (FDS) system consists of distributed smoke detection and portable $\mathrm{CO}_{2}$ fire suppression bottles (PFE). The smoke detector is based on photoelectric technology and detects smoke through increased levels of obscuration compared to normal atmosphere background readings. It is a simple design and proven reliable, but is somewhat susceptible to false alarm due to dust accumulation. The $\mathrm{CO}_{2}$ suppressant was chosen over other alternatives due to its effectiveness and ability to be easily removed by the atmosphere revitalization system. Because $\mathrm{CO}_{2}$ can reach toxic levels if large volumes of suppressant are released, the $I S S$ credible fire zones must be partitioned into smaller volumes capable of being suppressed with a single $6 \mathrm{lb}$. release of $\mathrm{CO}_{2}$. Open cabin fires not able to be suppressed by the PFE must be isolated and depressurized.

\section{E. Atmosphere Control and Supply}

Components making up the Atmosphere Control and Supply (ACS) system on-board the ISS are high pressure oxygen and nitrogen storage tanks for makeup gas, total pressure sensors, a vent and relief valve assembly, and a pressure control assembly. Oxygen and nitrogen lines are distributed throughout the station to supply the individual elements and equipment users. Emergency oxygen is available via the use of portable breathing apparatus which can be comnected to the oxygen supply system. This system is highly effective, but must currently be resupplied either via tank changeout or by repressurization from the Shuttle Orbiter via an oxygen compressor assembly (ORCA).

\section{Candidate Improvements to ISS Technologies}

Given the strengths and drawbacks of the ISS technologies from the previous discussion, below are some candidate areas of improvements that have been identified by MSFC and Johnson Space Center (JSC) Space Station ECLSS personnel. Thoughts are loosely organized according to primary benefit - reduce resupply and consumables, increase robustness/life/reduce complexity, or reduce mission vehicle resource requirements. It is recognized that there are relationships and trades between these various factors, any of which may be weighted more heavily depending on specific mission requirements. 


\section{A. Reduction of Resupply and Consumables}

Even as resupply availability and capability to the ISS is a finite resource, one made even more constrained if there is a loss of one of the cargo vehicles (Shuttle, Progress, Automated Transfer Vehicle (ATV), HII Transfer Vehicle (HTV)), as human spaceflight reaches further and prolonged destinations such as the moon and Mars the reliance on resupply must be minimized. Resupplied items for ECLSS can include makeup atmosphere gases, water, replacement parts for limited life and consumable items such as filters and pumps. On ISS, these items represent a significant yearly upmass penalty, a parameter weighted heavily in Advanced Life Support technology and system trades. The following are several areas where improvements to the baseline ISS ECLSS in this area can possibly be made.

\section{Greater closure of the air/water loop-minimize losses}

In the baseline ISS ECLSS, $\mathrm{CO}_{2}$ and $\mathrm{H}_{2}$ are vented overboard from the resulting carbon dioxide removal and oxygen generation processes. The addition of a carbon dioxide reduction system is a key missing link to close the air loop and recover precious water for crew consumption. The ISS ECLSS is scarred to accept the addition of a Sabatier $\mathrm{CO}_{2}$ Reduction Assembly (CRA) which would generate methane and water from the waste $\mathrm{CO}_{2}$ and $\mathrm{H}_{2}$. This system would recover $2000 \mathrm{lb}$ /year of water for a crew of four and is currently at a development TRL of 5 . Because there is not enough stoichiometric hydrogen to reduce all the metabolic carbon dioxide with the Sabatier reaction, the Sabatier CRA alone cannot completely close the oxygen loop. An additional technology which either pyrolyzes the resulting methane to solid carbon and hydrogen, or another carbon dioxide reduction technology such as a Bosch reactor, must be utilized. These technologies are currently at an estimated TRL between 1 and 4 , with significant development remaining to prove whether they would trade favorably over simply resupplying the additional water not recovered.

While the planned ISS Water Recovery System is $93 \%$ efficient in recovering potable water from waste water (an average between the $85 \%$ efficient urine processor and $100 \%$ efficient potable water processor), there is room for improvement in the amount of water recovered from the urine brine via a brine post-processor. A key challenge for the brine processor is how to effectively handle the solids that will precipitate out of solution as more water is evaporated. Technologies such as air evaporation appear to be attractive, as well as a system that may be able take advantage of partial gravity for a lunar/Mars base application.

\section{Decrease dependence on expendable beds}

The Trace Contaminant Control System's expendable charcoal and post-sorbent beds are not regenerated in place and must be periodically replaced. A regenerable adsorbent and catalyst substrate that is amenable to in-flight maintenance and addresses issues associated with pellet bed size attrition is emerging as a viable improvement solution. Development of metallic, short-channel length monolith substrates suitable for use in the thermal catalytic oxidizer has been in progress since 1994.,5 More recently, the metallic substrate has been adapted to adsorbent media and initial findings indicate a nearly $70 \%$ reduction in trace contaminant control adsorbent bed size with modest power consumption for regeneration. ${ }^{6}$ Near-term, the catalytic oxidation application of this substrate has achieved a TRL of 6 and a preliminary design has been formulated for retrofit into the ISS's TCCS. ${ }^{7}$

The ISS Water Processor Assembly relies on expendable multifiltration beds to partially remove the contaminant load in the wastewater. In addition, an ion exchange bed is required to remove products of oxidation from the volatile removal assembly reactor. The associated resupply requirement is equivalent to $1040 \mathrm{lb} / \mathrm{year}$. It is anticipated that significant reductions can be achieved over the baseline by employing a more efficient catalytic oxidizer in the cabin air system which would remove the bulk of the atmosphere contaminant load upstream of the condensing heat exchanger, thus reducing the load remaining for the water recovery system.

Although the current service life analysis for the ISS cabin air bacteria filter elements predicts a replacement interval of 2 years versus the initially expected 1 year period ${ }^{8}$, further improvements in particulate matter filtration may be required for extended operation of a habitat ventilation system beyond low earth orbit. The challenge will be to minimize the weight penalty associated with an expendable filter media, or to devise an innovative method of separating particulates from air without the use of expendable media.

\section{High Pressure Oxygen Supply}

The high pressure oxygen gas storage system onboard ISS primarily supports EVA and if necessary provides makeup metabolic oxygen. When depleted, these tanks must either be replaced or repressurized. Repressurization from the Shuttle's 900 psi cryogenic oxygen storage system to the ISS high pressure tank storage pressure of 2400 psi requires an intermediate oxygen recharge compressor assembly (ORCA). The ORCA has a limited life due to wearing of its pumping diaphragms. Improvements to the ORCA to extend its life and improve pumping capacity would minimize the impact of launch of replacement parts. Alternatively, a high pressure water electrolysis system capable of generating oxygen at 2400 psi directly from an ambient pressure water source would provide additional flexibility without the need of a separate oxygen compressor. It is anticipated that the atmosphere control system for 
an exploration vehicle or habitat would have a similar requirement for a ready and reliable backup oxygen storage system.

\section{B. Increase Robustness/Reduce Complexity}

Experience has shown that the more complex a technology, the more prone it will be toward failures. The number of major components drives the number of associated sensors and effectors necessary to control and monitor the system and meet safety fault tolerance and failure isolation requirements typical for manned spaceflight hardware. The following are specific lessons leamed from ISS ECLSS development history and associated recommendations for future improvements.

\section{Sensitivity to Particulates}

A frequent contributor to ISS on-orbit problems has been sensitivities of various components to particulate contamination. The generation, release, and migration of particulate matter are often substantially different in microgravity than in 1-g. If not properly accounted for in component design, unexpected problems can occur in flight. The following are some examples from iSS ontorbit experience.

Failure of the CDRA air-save pump, bed check valve, and selector valve resulted from small releases of sorbent material from the packed beds. The failure manifested itself on orbit where the sorbent material was not constrained by gravity and worked its way into downstream components. As a temporary solution, in-line filters have been installed in the CDRA on-orbit to trap particulates and protect downstream components. A redesign of the sorbent bed containment system is underway as a permanent solution to be implemented on the second CDRA unit to be flown on Node 3.

A similar failure occurred on the technology risk mitigation flight experiment of the ISS Water Processor Assembly's Volatile Removal Assembly (VRA) on STS-89. In that experiment, particulates generated within the VRA's packed-bed catalytic reactor migrated on-orbit to a pressure regulator and a membrane phase separator downstream of the reactor, causing premature shutdown of the experiment. Post-flight investigation further revealed that migration of catalyst fines appeared to have occurred during pre-flight ground testing but went unnoticed due to the effects of gravity which masked the effects.

The unanticipated accumulation of a thick layer of lint on an intermodule ventilation fan's inlet screen led to degraded flow on-orbit. The buildup was due to passage of small lint through the subsystems inlet filter screens and subsequent aggregation into larger clumps which caused the flow blockage. As a result of the lack of fine particle settling in microgravity both the size distribution and quantity of particulate load differ substantially from that upon which the ventilation system's inlet screen was designed. To resolve the problem on ISS, a duct lint filter will be installed.

Ambient dust has caused the Node 1 smoke detector to trigger false positive alarms. Although the detector is located behind the cabin fan's inlet screen, dust is accumulating necessitating periodic cleaning by the crew. Characterization and accounting for background dust levels must be accounted for, or an alternate, dustinsensitive detector is needed.

Several failures have occurred during ground assembly and testing of the ISS Water and Urine Processors, due to random particulate contamination of solenoid, check, or relief valves. Tightly-designed clearances in these components make them more susceptible to even minute particles generated during the manufacturing process. Stricter cleaning processes have been implemented, along with installation of in-line filter protection. In the future, trades between robustness of these types of components versus precision performance must be made taking into consideration the likely particulate loading operating conditions.

\section{Condensing Heat Exchanger Coating Degradation}

The cabin condensing heat exchanger is designed with coatings to provide hydrophilic and biocidal properties. Liquid carryover occurring in a set of heat exchangers in the U.S. Lab is believed to be due to a failure of the hydrophilic coating, which is also believed to be releasing solids into the condensate stream when the heat exchanger experiences frequent cycles from wet to dry. These solids end up fouling the downstream condensate separator, check valves or filters. Degradation of the hydrophilic coatings through exposure to contaminants or operating cycles needs to be accounted for in future systems designs.

3. Less complex, more reliable sensors

While the ISSS mass spectrometry-based atmosphere monitor for major constituents provides stable, repeatable measurements, it has life limitations associated with its ion pump and also requires a vacuum interface for startup. It is a relatively large and complex piece of equipment for monitoring of only six specific constituents. Simple, optically-based sensors are emerging that can do the job with no need to maintain vacuum. Recent work in this area has found fast-diode laser oxygen sensors, solid state infrared carbon dioxide sensors, and thin-film capacitive humidity sensors to be viable candidates for further consideration. ${ }^{10}$ Also, an array of single-analyte sensors targeted 
at specific trace species such as ammonia, formaldehyde, carbon monoxide, and oxides of nitrogen would greatly enhance overall environmental monitoring.

Unlike open-loop spacecraft propellant supply and management systems which are charged with high purity, very low dewpoint hydrogen prior to a mission, regenerative life support technologies generate and have to maintain hydrogen containing traces of other gases and with high dewpoints. Stable, highly accurate hydrogen measurements in the range required for reliable safety controls (typically $1 \%$ hydrogen in oxygen) are difficult to achieve with today's technologies. Relatively short calibration intervals require frequent on-orbit replacement of these sensors and represent a significant logistics penalty. Stable, reliable sensors for this type of application are needed to improve the current state-of-the-art for future systems.

\section{Water Phase Organic Oxidation}

The ISS Water Processor Assembly's catalytic reactor employs a noble metal catalyst that has been shown to be effective at oxidizing a wide range of trace organic contaminants in water in the presence of gaseous oxygen at elevated temperature ( 275 F). However, system complexity is increased by the need to manage two-phase fluid flow through a nacked bed reactor in microgravity, by inherent power, packaging, and materials penalties associated with elevated temperature operation, by the need to protect the catalyst from potential poisons through the use of upstream expendable beds, and by the practical limit of the reactor's oxidation capacity. A robust, single-phase, low or ambient temperature catalyst with high oxidation capacity would be an attractive alternate which would both simplify the process and reduce resource requirements. In-situ generation of the oxidant from the process water itself could also provide benefit over supply from stored tanks.

5. Passive Phase Separation

Membrane phase separators are an attractive choice for advanced life support designs due to their attributes of no moving parts and low power consumption. However, surface properties of the membrane which are required for proper functioning (hydrophilic or hydrophobic) are often susceptible to particulate, microbial, or organic contamination, further aggravated in microgravity. Liquid condensation on the gas side of hydrophobic membranes is also problematic and requires careful control of thermal gradients and dilution with dry gas. Alternate passive phase separators which are tolerant of these conditions would be attractive.

\section{Non-hazardous Urine Pretreatment}

Pretreatment with chemical additives is required to chemically and microbially stabilize urine prior to processing. Current pretreatment formulas rely on the use of toxic and acidic reagents which drive system design in terms of hazardous fluid containment, seal design for leakage control, and materials compatibility. Pretreatment formulations which are based on nontoxic, neutral reagents would simplify urine processor designs. A method of generating these pretreatment reagents in-situ from the constituents of urine itself would offer an added advantage.

\section{Reduce Resource Requirements}

In addition to reducing resupply requirements and increasing robustness, systems which minimize consumption of vehicle resources such as initial launch weight, volume, power, and crew time for maintenance trade more favorably, especially if these resources are mission-limited, which has historically been the case.

By combining subsystems which have traditionally been treated separately, efficiencies in packaging, thermal cooling, and power can be obtained. Care must be taken, however, to ensure proper system redundancy is accounted for since, for example, a problem with one component can impact multiple life support functions at the same time. Integrated, regenerative, carbon dioxide, humidity control, and trace contaminant control systems appear attractive for next generation systems. Coupling the trace contaminant control and carbon dioxide removal functions into a single atmosphere revitalization function along with a membrane-based process air drying stage has been studied." This concept uses the monolithic substrate for both the trace contaminant control and carbon dioxide removal stages. The resulting system is fully regenerable, requires no process air pre-processing, and can provide high-purity $\mathrm{CO}_{2}$ to a Sabatier reactor.

It stands to reason that the previous discussions on reduction of expendables would also improve on-orbit volume for spare component storage and crew time for replacement. Some of the suggested improvements in robustness would also reduce crew time for repair, and in some cases reduce power requirements. These factors are naturally related.

\section{General Lessons Learned from ISS Development}

Several more general, but key lessons have been learned from development of the baseline ISS ECLSS that should be considered in future development efforts. One of these lessons is that even seemingly small design

6 
changes made in the spirit of "making better" can often lead to unforeseen, negative consequences. In one example, the development pressure sensor in the Urine Processor Assembly was manufactured from stainless steel and exhibited stable performance over years of testing. A change to inconel was selected for the flight system in order to improve robustness in the pretreated urine environment. An unexpected drift in the flight sensor reading was detected and attributed to offgassing of the inconel in the sensor's reference chamber. Similar drifts were experienced in pressure sensors made from inconel used in the oxygen generation assembly. A change back to the original stainless steel sensor design is being made to both assemblies.

When a new technology is being developed, great attention is naturally given to the major components within the heart of the system. However, in the course of flight hardware development for the ISS Regenerative ECLSS project, many times it has been the small, ancillary components that have presented the greatest challenges. Some examples are check valves which required mods to springs, poppets, and valve bore surfaces to perform at the required low cracking pressures, leaking relief valves which required process improvements in the seal molding, faulty valve position indicators, water storage tank quantity sensors which required redesign of their potentiometers to meet cycle life requirements, and solenoid valves subject to "stiction" requiring redesign to increase solenoid force. In development of the next generation ECLSS, care must be taken to thoroughly address all ancillary components necessary for the complete subsystem so that these kinds of problems are not discovered so late that they significantly impact flight hardware delivery schedules.

Another normal pitfall is the tendency to underestimate the effort and time involved in taking a subsystem from what appears to be a fairly mature development stage (TRL-5 or 6) to flight hardware. We optimistically believe that most problems have been solved in laboratory-type development testing and that the remaining task to produce flight hardware is relatively straightforward from that point. Also, it is human nature to project rather successoriented schedules, neglecting to account for problems which, depending on their severity, may cause significant setbacks and rework. Depending on the corrective action, new long-lead parts may need to be ordered and the design-analysis-drawing review-manufacturing cycle repeated. When one-of-a-kind, protoflight systems are built to save cost over the traditional, qualification/flight article approach, additional schedule and cost risk is borne by the project.

Finally, when trading subsystems at lower TRL's, the additional burden of added sensors and effectors necessary to meet safety critical and catastrophic hazard control requirements must be accounted for. Experience has shown that a subsystem typically grows by at least $50 \%$ from the initial functional schematic to that necessary to meet hazard controls. Depending on the inherent potential hazards of a particular technology, the impact could range from relatively small to very significant (e.g. packaging of the entire oxygen generation assembly's cell stack ORU within a large pressure dome). Creative ways to manage hazards while minimizing the burden of additional components, which adds another layer of complexity and decreases robustness, becomes even more necessary as we plan for missions beyond low Earth orbit.

\section{Conclusion}

Advances in Life Support System technologies can be categorized as either "evolutionary" or "revolutionary". While higher-risk, potentially higher payoff, revolutionary ideas should be pursued in parallel, evolutionary advances over the current state of the art offer a more logical starting point for the first exploration spirals. The ISS baseline ECLSS and associated lessons learned through development and on orbit experience has been discussed along with suggested evolutionary areas of improvement for next generation systems.

\section{References}

'Perry, J.L., Cole, H.E., Cramblitt, E.L., El-Lessy, H.N., Manuel, S., and Tucker, C.D., "Post-Flight Sampling and Loading Characterization of Trace Contaminant Control Subassembly Charcoal", SAE 2003-01-2487, SAE 2003.

${ }^{2}$ Murdoch, K., Smith, F., Perty, J., and Green S., “Assessment of Technology Readiness Level of a Carbon Dioxide Reduction Assembly (CRA) for Use on International Space Station", SAE 2004-01-2446, SAE 2004.

${ }^{3}$ Hutchens, C.F. and Graves, R., "Results of the Vapor Compression Distillation Flight Experiment (VCD-FE)", SAE 200401-2450, SAE 2004.

${ }^{4}$ Carter, R.N., Bianchi, J.F., Pfefferle, W.C., Roychoudhury, S., and Perry, J., "Unique Metal Monolith Catalytic Converter for Destruction of Airborne Trace Contaminants", SAE 972432, SAE 1997. 
${ }^{5}$ Perry, J.L., Frederick, K.R., Carter, R.N., and Tatara, J.D., "Performance Demonstration of a Metal Monolith Catalytic Converter for Trace Contaminant Control", NASA/TM-1999-209262, NASA MSFC, April 1999.

"Roychoudhury, S., Walsh, D., and Perty, J., "Microlith Based Sorber for Removal of Environmental Contaminants", SAE 2004-01-2442, SAE 2004.

${ }^{7}$ Evans, T., "Design, Fabrication, and Test of a Developmental Metal Monolith Catalytic Converter for Trace Contaminant Control", NAS8-40369-5152. Precision Combustion, Inc., North Haven, CT, August 2000.

${ }^{8}$ Thompson, D., "ISS ECLSS Expendable Service Life Improvements", SAE 2003-01-2492, SAE 2003.

${ }^{9}$ Chiramonte, F.P., and Joshi, J.A., "Workshop on Critical Issues in Microgravity Fluids, Transport, and Reaction Processes in Advanced Human Support Technology", NASA/TM-2004-212940. February, 2004, pp. 14-22.

${ }^{10}$ Tatara, J.D., and Perry, J.L., "Spacecraft Cabin Atmosphere Major Constituent Monitoring Using Off-the-Shelf Techniques", SAE 2004-02-2541, SAE 2004.

"Mulloth, L.M., Perry, J.L., and LeVan, M.D., "System Design for Air Revitalization in Next Generation Crewed Spacecraft", SAE 2004-01-2373, SAE 2004. 